\title{
Topical Rapamycin Therapy to Alleviate the Cutaneous Manifestations of Tuberous Sclerosis Complex
}

\section{A Double-Blind, Randomized, Controlled Trial to Evaluate the Safety and Efficacy of Topically Applied Rapamycin}

Mary Kay Koenig, ${ }^{1,2}$ Adelaide A. Hebert, ${ }^{2,3,4}$ Joan Roberson, ${ }^{2}$ Joshua Samuels, ${ }^{2,5,6}$ John Slopis, ${ }^{7,8,9}$ Audrey Woerner ${ }^{10}$ and Hope Northrup ${ }^{2,11}$

1 Division of Child \& Adolescent Neurology, Department of Pediatrics, University of Texas Medical School at Houston, Houston, TX, USA

2 Tuberous Sclerosis Complex Center of Excellence, Division of Medical Genetics, University of Texas Medical School at Houston, Houston, TX, USA

3 Department of Dermatology, University of Texas Medical School at Houston, Houston, TX, USA

4 Department of Pediatrics, University of Texas Medical School at Houston, Houston, TX, USA

5 Division of Pediatric Nephrology, Department of Pediatrics, MD Anderson Cancer Center \& University of Texas Medical School at Houston, Houston, TX, USA

6 Division of Renal Diseases/Hypertension, Department of Internal Medicine, MD Anderson Cancer Center \& University of Texas Medical School at Houston, Houston, TX, USA

7 Department of Neuro-Oncology, MD Anderson Cancer Center, Houston, TX, USA

8 Department of Pediatrics, MD Anderson Cancer Center, Houston, TX, USA

9 Neurofibromatosis Center, MD Anderson Cancer Center, Houston, TX, USA

10 Division of Medical Genetics, Department of Pediatrics, Harvard Medical School, Boston, MA, USA

11 Division of Medical Genetics, Department of Pediatrics, University of Texas Medical School at Houston, Houston, TX, USA

Background and Objectives: Facial angiofibromas are disfiguring facial lesions, present in up to $80 \%$ of patients with tuberous sclerosis complex. Recent elucidation of the complex cell signaling pathways that are disrupted in tuberous sclerosis indicates that rapamycin may be successful in alleviating the appearance of these lesions. The objectives of the current study were to evaluate the safety of topically applied rapamycin in patients with tuberous sclerosis complex and to determine its potential effectiveness in treatment of facial angiofibromas.

Patients and Methods: The study was a prospective, randomized, doubleblind, placebo-controlled study performed at the University of Texas Health Science Center at Houston. Study subjects were recruited from the patient populations at the University of Texas Tuberous Sclerosis Center of Excellence. All subjects were over the age of 13 years and had a diagnosis of 
tuberous sclerosis complex. Subjects were excluded if they were using any form of rapamycin or if they were pregnant. Study subjects applied the study product to their facial angiofibromas nightly for a duration of 6 months. The investigational product contained one of three doses of rapamycin compounded with Skincerity ${ }^{\circledR}$ : (i) no rapamycin; (ii) $1 \mathrm{mg}$ of rapamycin per $30 \mathrm{cc}$ $(0.003 \%)$; or (iii) $5 \mathrm{mg}$ of rapamycin per $30 \mathrm{cc}(0.015 \%)$. Plasma rapamycin concentrations were measured monthly to test for systemic absorption. Complete blood counts were performed monthly to test for anemia, neutropenia, or thrombocytopenia. Upon completion of the trial, subjects were asked if the formulation had improved the appearance of their facial angiofibromas.

Results: Twenty-three subjects completed the study. There was no detectable systemic absorption of rapamycin (all blood concentrations were $<1.0 \mathrm{ng} / \mathrm{mL}$ ). There were no significant changes in white blood cell, red blood cell, or platelet counts. Seventy-three percent of subjects in the treatment arms versus $38 \%$ of subjects in the placebo arm reported a subjective improvement in the appearance of their facial angiofibromas.

Conclusion: The application of low-dose topical rapamycin $(0.003-0.015 \%)$ to the face can safely decrease the appearance of facial angiofibromas in patients with tuberous sclerosis complex.

Trial Registration: ClinicalTrials.gov Identifier: NCT01031901

Tuberous sclerosis complex (TSC) is an autosomal-dominant genetic disorder characterized by the formation of benign tumors in multiple organ systems. Facial angiofibromas appear as red or pink papules over the central face, especially on the nasolabial folds, cheeks, and chin, ${ }^{[1]}$ in people with TSC. Lesions arise in early childhood and are present in up to $80 \%$ of TSC patients. ${ }^{[1,2]}$ In some patients, the lesions become confluent and can result in severe disfigurement. Although multiple treatments have been developed to alleviate the appearance of facial angiofibromas - curettage, cryosurgery, chemical peels, dermabrasion, shave excisions, and laser therapy ${ }^{[3-8]}$ - these are uncomfortable and need to be repeated at periodic intervals to treat recurrence. ${ }^{[9]}$

In patients with TSC, the mammalian target of rapamycin (mTOR) is aberrantly activated in fibroblast-like cells located within the dermal layer of the skin. These cells produce an epidermal growth factor, epiregulin, which stimulates epidermal cell proliferation. ${ }^{[10]}$ Epidermal cells are produced at a faster rate than the ability to slough the dead cells from the skin surface. ${ }^{[11]}$ This overproduction of skin cells, in conjunction with angiogenesis, results in the initial appearance and continued progression of facial angiofibromas over time.

Recent elucidation of the complex signaling relationship between the tuberous sclerosis 1 (TSC1) and tuberous sclerosis 2 (TSC2) gene products and $\mathrm{mTOR}$ has led to an explosion of research related to the use of mTOR inhibitors, such as rapamycin, in TSC. These mTOR inhibitors are showing promise in treating multiple tumor types, including renal angiomyolipomas (AMLs), sub-ependymal giant cell astrocytomas (SEGAs), and lymphangioleiomyomatosis (LAM). ${ }^{[2-15]}$

Rapamycin is a naturally occurring antifungal macrolide, first isolated from Streptomyces hygroscopicus in 1965. Rapamycin binds with high specificity to mTOR, and binding results in inhibition of mTOR activity and ultimately in downregulation of cell growth. ${ }^{[16]}$ Rapamycin 
has a molecular weight of 914.2 grams $/ \mathrm{mol}$, allowing for its absorption through the superficial layers of the epidermis to the deep dermal layer implicated in the development of facial angiofibromas.

The primary goal of this study was to evaluate the safety of topical rapamycin $(0.003 \%$ and $0.015 \%$ ) in patients with TSC. The secondary goal of this study was to evaluate the efficacy of the topical product for treatment of facial angiofibromas.

\section{Methods}

\section{Patient Selection}

After approval was obtained from the institutional review board at the University of Texas Health Science Center (UTHSC) at Houston, study subjects were recruited from the patient population at the Tuberous Sclerosis Center of Excellence at the University of Texas Medical School at Houston from January 2010 through August 2010. All subjects were over the age of 13 years and had a clinical diagnosis of tuberous sclerosis complex. ${ }^{[17]}$ Subjects were excluded if they were currently pregnant, were using oral rapamycin, or had any form of immune dysfunction. After completing an informed consent document, willing participants who satisfied the inclusion and exclusion criteria (table I) were enrolled in the study. The study participants provided demographic data, including age, sex, and race, during the initial interview. Race/ethnicity was defined by the participants.

\section{Protocol Summary}

Upon enrollment, subjects were randomized and provided with a bottle of the investigational product. The investigational product contained one of three doses of rapamycin compounded with Skincerity ${ }^{\circledR}$ : (i) no rapamycin; (ii) $1 \mathrm{mg}$ of rapamycin per $30 \mathrm{cc}(0.003 \%)$; or (iii) $5 \mathrm{mg}$ of rapamycin per $30 \mathrm{cc}(0.015 \%)$. Subjects applied a thin coating of the investigational product to the skin directly over the treatment area every evening for 6 months. The product was allowed to air dry for 60 minutes following application and was
Table I. Inclusion and exclusion criteria for study participation

\section{Inclusion criteria}

- Male and female patients aged 13 years and older

- Patients with a clinical diagnosis of tuberous sclerosis complex ${ }^{[17]}$ and visible facial angiofibromas

- Patients willing and able to comply with the requirements of the study

\section{Exclusion criteria}

- Sexually active women of childbearing potential not using adequate contraception

- Pregnant women or nursing mothers

- Patients with immune dysfunction or receiving any form of immunosuppression

- Patients currently receiving therapy with rapamycin

- Patients currently participating, or having participated within the last 30 days, in any clinical trial involving an investigational drug product

- Patients with known hypersensitivity to any component of the study product

removed by gentle washing in the morning. Subjects were monitored monthly in the study clinic, where blood was drawn to measure serum rapamycin concentrations, the study product was examined, and photographs were taken. The study product was replenished as needed.

Rapamycin concentrations were analyzed at the University of Texas Medical School at Houston rapamycin laboratory, using an Architect 11000 analyzer (Abbott Laboratories, Abbott Park, IL, USA).

The investigational product was manufactured at no cost by Biomedical Development Corporation (San Antonio, TX, USA) by combining sirolimus with Skincerity ${ }^{\circledR}$. The company randomized the investigational product. The researchers and study subjects were not provided with the randomization information until all data had been collected. Biomedical Development Corporation had no role in the design of the trial; in the collection, analysis, or interpretation of the data; or in the writing of this manuscript. The authors vouch for the accuracy and completeness of the reported data.

\section{Data Collection and Statistical Methods}

Upon completion of the trial, subjects were asked if they "got better on the treatment", if 
they "got worse on the treatment", or if "the treatment made no difference". Statistical analysis was performed using a two-sided Fischer's exact test. At each study visit, blood was drawn to measure serum rapamycin concentrations and complete blood counts to assess for systemic absorption of the study product.

\section{Results}

\section{Patient Demographics}

A total of 28 patients met the criteria for enrollment in the study: 15 men (54\%) and 13 women (46\%). The mean patient age at the time of study enrollment was 23 years; $72 \%$ of the patients were non-Hispanic Whites, $7 \%$ were Hispanic, $10 \%$ were African American, 7\% were Asian, and 4\% were Native American (table II). No subject who was invited to participate refused. Two subjects withdrew from the study, secondary to discomfort with product application (a burning sensation with application). Two subjects were withdrawn by the investigators, secondary to poor compliance with study protocols. One subject was withdrawn following prolonged hospitalization unrelated to the study product. Twenty-three subjects completed the entire study protocol.

Table II. Patient demographic data

\begin{tabular}{|c|c|c|c|c|}
\hline \multirow[t]{2}{*}{ Characteristic } & \multirow{2}{*}{$\begin{array}{l}\text { Placebo } \\
\text { (n) }\end{array}$} & \multicolumn{2}{|c|}{ Rapamycin (n) } & \multirow{2}{*}{$\begin{array}{l}\text { Total } \\
\text { (n [\%]) }\end{array}$} \\
\hline & & High-dose & Low-dose & \\
\hline \multicolumn{5}{|l|}{ Age (years) } \\
\hline $13-18$ & 3 & 2 & 3 & 8 [29] \\
\hline $19-24$ & 3 & 3 & 5 & 11 [39] \\
\hline $25-30$ & 1 & 1 & 0 & $2[7]$ \\
\hline$>30$ & 3 & 3 & 1 & $7[25]$ \\
\hline \multicolumn{5}{|l|}{ Sex } \\
\hline Male & 4 & 6 & 5 & $15[54]$ \\
\hline Female & 6 & 3 & 4 & $13[46]$ \\
\hline \multicolumn{5}{|l|}{ Race } \\
\hline White, non-Hispanic & 6 & 7 & 7 & 20 [72] \\
\hline White, Hispanic & 2 & 0 & 0 & $2[7]$ \\
\hline African American & 0 & 2 & 1 & $3[10]$ \\
\hline Asian & 1 & 0 & 1 & $2[7]$ \\
\hline Native American & 1 & 0 & 0 & $1[4]$ \\
\hline
\end{tabular}

\section{Rapamycin Concentrations}

Blood was drawn at each study visit to measure serum rapamycin concentrations. The limit of quantitation of this immunoassay was $1.0 \mathrm{ng} / \mathrm{mL}$, and no subject reached that concentration during treatment.

\section{Complete Blood Counts}

Complete blood counts were performed by Quest Laboratories. No subject demonstrated a significant change in the white blood cell count, hemoglobin level, or platelet count during treatment.

\section{Lesion Appearance}

Of the 23 subjects who completed the study, $73 \%$ in the treatment arms reported that "they got better on the treatment", versus $38 \%$ of subjects in the placebo arm (figure 1) $[\mathrm{p}=0.18]$. Twenty-seven percent of subjects in the treatment arms reported that "the treatment made no difference", versus $62 \%$ of subjects in the placebo arm. No subject reported that they "got worse on the treatment". There was no statistically significant difference in the response between subjects on high-dose and low-dose treatment.

\section{Serious Adverse Events}

Among the study subjects, a serious single adverse event occurred in a patient in the low-dose arm of the study. This subject aspirated during a seizure and developed pneumonia, which progressed to septic shock. His rapamycin concentrations were undetectable at the time of hospital admission, and he was immediately withdrawn from the study. His illness required prolonged hospitalization, but he has since made a full recovery.

\section{Discussion and Conclusion}

TSC is a genetic disorder affecting 1 in 6000 individuals worldwide. It is characterized by abnormal skin pigmentation and tumor formation in multiple organ systems. Facial angiofibromas are benign skin tumors found on the faces of patients with TSC, and the angiofibromatous lesions appear as red or pink papules distributed over the central face, most notably on the nasolabial folds, 
a

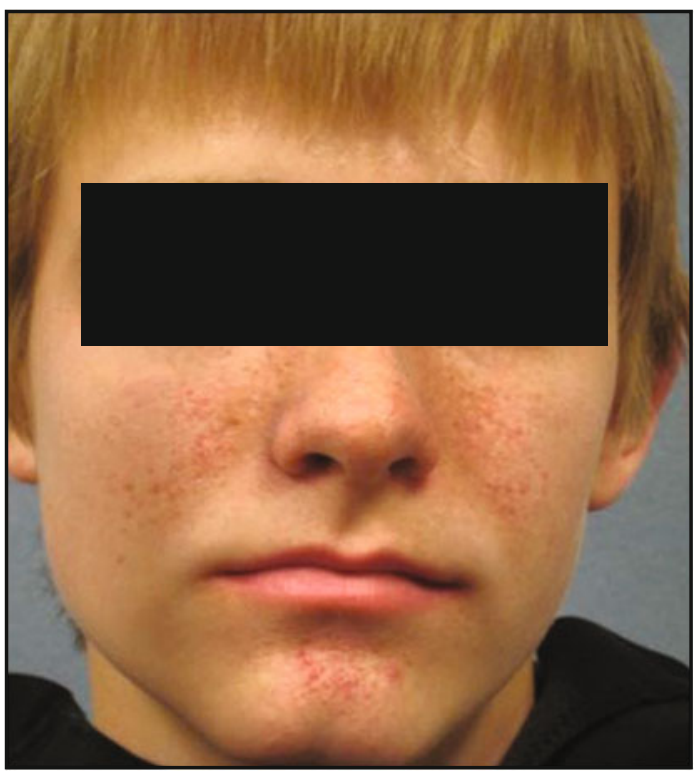

b

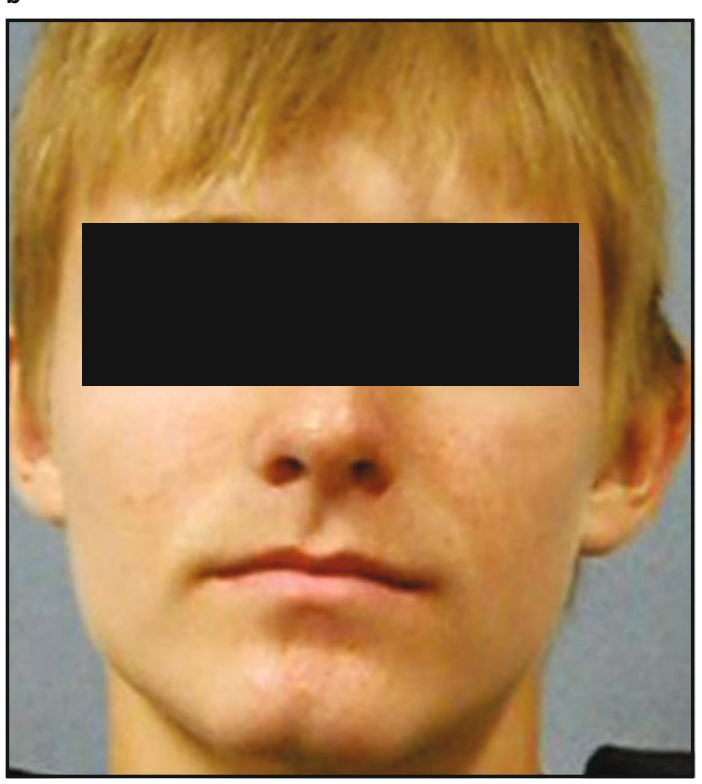

Fig 1. Study subject (a) prior to therapy and (b) following 6 months of treatment with topical rapamycin. The subject reported complete resolution of his facial angiofibromas.

cheeks, and chin. Lesions appear in early childhood and are present in up to $80 \%$ of TSC patients, creating considerable cosmetic morbidity.
Since the initial descriptions of facial angiofibromas in the 19th century, multiple treatments have been developed, attempting to alleviate the appearance of these lesions, including curettage, cryosurgery, chemical peels, dermabrasion, shave excisions, and laser therapy. Although the majority of these treatments are initially effective, they are uncomfortable, and over time the lesions recur.

Recent case reports and small case series have demonstrated that a topical rapamycin formulation might be efficacious, ${ }^{[18-27]}$ but prior reports have consisted of small series without placebo arms. The primary goal of this study was to determine whether our topical formulation of rapamycin was safe for topical use in the treatment of facial angiofibromas in patients with TSC. The study was designed to see if application of the investigational product resulted in detectable systemic absorption of the rapamycin. The secondary goal of this study was to evaluate whether the topical product decreased the appearance of the facial angiofibromas after 6 months of usage, as self-reported by the subjects. Twenty-three study subjects applied either a placebo or the investigational product nightly to their lesions for 6 months. The study results showed that there were no serious adverse events related to the study product and there was no detectable systemic absorption of the rapamycin during the study period. This study was not powered to reach statistical significance, and although it did not, almost twice as many subjects in the combined treatment arms reported subjective improvements $(73 \%)$ when compared with the placebo arm $(38 \%)$. The observed effect could result from a treatment effect of the vehicle itself, but these results suggest that topical application of our study product is effective in improving the appearance of facial angiofibromas in people with TSC. Future studies will include more detailed monitoring of efficacy, including standardized photography and monthly quality-oflife questionnaires.

\section{Acknowledgments}

This study was supported in part by the Society for Pediatric Dermatology, Cheniere Energy, Inc., the Sponsors of 
Kirk and Meg Gentle of the Cheniere Race Across America Team, the University of Texas Medical School at Houston Department of Pediatrics, and the University of Texas Tuberous Sclerosis Center of Excellence at the University of Texas Medical School at Houston. The sponsors had no role in the design and conduct of the study; in the collection, analysis, or interpretation of data; or in the preparation, review, or approval of the manuscript. The authors have no relevant financial or conflicts of interest to disclose. We are indebted to Dr. Laura Lester and Dr. Laura Marusinec for their assistance in this clinical trial. We thank Biomedical Development Corporation for their role in the production of the topical study product.

\section{References}

1. Schwartz RA, Fernandez G, Kotulska K, et al. Tuberous sclerosis complex: advances in diagnosis, genetics, and management. J Am Acad Dermatol 2007; 57: 189-202

2. Kane Y. The "bumps" on my face. J Am Acad Dermatol 2004; 51: S11-2

3. El-Musa KA, Shehadi RS, Shehadi S. Extensive facial adenoma sebaceum: successful treatment with mechanical dermabrasion: case report. Brit J Plast Surg 2005; 58: 1143-7

4. Finch TM, Hindson C, Cotterill JA. Successful treatment of adenoma sebaceum with the potassium titanyl phosphate laser. Clin Exp Dermatol 1998; 23: 201-3

5. Hori K, Soejima K, Nozaki M, et al. Treatment of facial angiofibromas of tuberous sclerosis using cultured epithelial autografts. Ann Plast Surg 2006; 57: 415-7

6. Kaufman AJ, Grekin RC, Geisse JK, et al. Treatment of adenoma sebaceum with the copper vapor laser. J Am Acad Dermatol 1995; 33: 770-4

7. Papadavid E, Markey A, Bellaney G, et al. Carbon dioxide and pulsed dye laser treatment of angiofibromas in 29 patients with tuberous sclerosis. Brit J Plast Surg 2002; 147: $337-42$

8. Verheyden $\mathrm{CN}$. Treatment of the facial angiofibromas of tuberous sclerosis. Plast Reconstr Surg 1996; 98: 777-83

9. Bittencourt RC, Huilgol SC, Seed PT, et al. Treatment of angiofibromas with scanning carbon dioxide laser: a clinicopathologic study with long-term follow-up. J Am Acad Dermatol 2001; 45: 731-5

10. Li S, Takeuchi F, Wang J, et al. Mesenchymal-epithelial interactions involving epiregulin in tuberous sclerosis complex hamaratomas. PNAS 2008; 105: 3539-44

11. Darling TN. Hamartomas and tubers from defects in harmartin-tuberin. J Am Acad Dermatol 2004; 51: S9-11

12. Bissler JJ, McCormack FX, Young LR, et al. Rapamycin for angiomyolipoma in tuberous sclerosis complex or lymphangioleiomyomatosis. New Engl J Med 2008; 358: 140-51

13. Franz DN, Leonard J, Tudor C, et al. Rapamycin causes regression of astrocytomas in tuberous sclerosis complex. Ann Neurol 2006; 59: 490-8

14. Koenig MK, Butler IJ, Northrup H. Regression of subependymal giant cells astrocytoma with rapamycin in tuberous sclerosis complex. J Child Neurol 2008; 23: 1238-9
15. Glasgow CG, Steagall WK, Taveira-DaSilva A, et al. Lymphangioleiomyomatosis (LAM): molecular insights lead to targeted therapies. Resp Med 2010; 104: S45-58

16. Paghdal KV, Schwartz RA. Sirolimus (rapamycin): from the soil of Easter Island to a bright future. J Am Acad Dermatol 2007; 57: 1046-50

17. Roach ES, DiMario FJ, Kandt RS, et al. Tuberous sclerosis consensus conference: recommendations for diagnostic evaluation. National Tuberous Sclerosis Association. J Child Neurol 1999; 14: 401-7

18. Foster RS, Bint LJ, Halbert AR. Topical $0.1 \%$ rapamycin for angiofibromas in paediatric patients with tuberous sclerosis: a pilot study of four patients. Australas J Dermatol 2012; 53: 52-6

19. Truchuelo T, Diaz-Ley B, Rios L, et al. Facial angiofibromas treated with topical rapamycin: an excellent choice with fast response. Dermatol Online J 2012; 18: 15

20. Wataya-Kaneda M, Tanaka M, Nakamura A, et al. A novel application of topical rapamycin formulation, an inhibitor of mTOR, for patients with hypomelanotic macules in tuberous sclerosis complex. Arch Dermatol 2012; 148: 138-9

21. DeKlotz CM, Ogram AE, Singh S, et al. Dramatic improvement of facial angiofibromas in tuberous sclerosis with topical rapamycin: optimizing a treatment protocol. Arch Dermatol 2011; 147: 1116-7

22. Salido R, Garnacho-Saucedo G, Cuevas-Asencio I, et al. Sustained clinical effectiveness and favorable safety profile of topical sirolimus for tuberous sclerosis-associated facial angiofibroma. J Eur Acad Dermatol Venereol. Epub 2011 Aug 11

23. Valeron-Almazan P, Vitiello M, Abuchar A, et al. Topical rapamycin solution to treat multiple facial angiofibromas in a patient with tuberous sclerosis. Actas Dermosifiliogr 2012; 103: 165-6

24. Mutizwa MM, Berk DR, Anadkat MJ. Treatment of facial angiofibromas with topical application of oral rapamycin solution $\left(1 \mathrm{mg} \mathrm{mL}^{-1}\right)$ in two patients with tuberous sclerosis. Br J Dermatol 2011; 165: 922-3

25. Wataya-Kaneda M, Tanaka M, Nakamura A, et al. A topical combination of rapamycin and tacrolimus for the treatment of angiofibroma due to tuberous sclerosis complex (TSC): a pilot study of nine Japanese patients with TSC of different disease severity. Br J Dermatol 2011; 165: 912-6

26. Kaufman McNamara E, Curtis AR, Fleischer Jr AB. Successful treatment of angiofibromata of tuberous sclerosis complex with rapamycin. J Dermatolog Treat 2012; 23: 46-8

27. Haemel AK, O'Brian AL, Teng JM. Topical rapamycin: a novel approach to facial angiofibromas in tuberous sclerosis. Arch Dermatol 2010; 146: 715-8

Correspondence: Assistant Professor Mary Kay Koenig, Department of Pediatrics, Division of Child and Adolescent Neurology, University of Texas Health Science Center, 6431 Fannin Street, Suite 3.153, Houston, TX 77030, USA.

E-mail: mary.k.koenig@uth.tmc.edu 